INPLASY

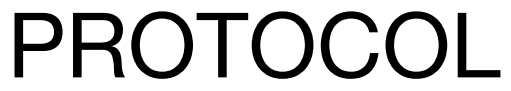

To cite: Liu et al. Potential link of neutrophil to lymphocyte ratio with pathological liver conditions in chronic hepatitis

$B$ patients: a meta-analysis. Inplasy protocol 202150020. doi:

10.37766/inplasy2021.5.0020

Received: 05 May 2021

Published: 05 May 2021

Corresponding author:

Qin Wang

Wangq@swmu.edu.cn

Author Affiliation:

Affiliated Hospital of

Southwest Medical University

Support: Southwest Medical

University.

Review Stage at time of this submission: Data analysis.

Conflicts of interest:

None declared.

\section{Potential link of neutrophil to lymphocyte ratio with pathological liver conditions in chronic hepatitis B patients: a meta-analysis}

\author{
Liu, XQ1; Ye, WX2 Fu, H3; Zhu, SR4; Fu, W5; Han, YS6; Wang, Q7.
}

Review question / Objective: The central white blood cell populations, neutrophils, and lymphocytes are involved in the pathophysiology of patients with chronic hepatitis B (CHB) infection. We thus performed a meta-analysis of studies investigating the potential link of neutrophil to lymphocyte ratio (NLR) with pathological liver conditions in CHB patients. Information sources: A comprehensive literature search was conducted by searching PubMed, EMBASE, Web of Science, Cochrane, the Chinese National Knowledge Infrastructure database, and the Wanfang online databases from inception to March 1, 2021. The search keywords were as follows: "neutrophil to lymphocyte ratio", "neutrophil lymphocyte ratio", "neutrophil-to-lymphocyte ratio", "neutrophillymphocyte ratio", "NLR", "hepatitis B", "chronic hepatitis B", "CHB", "hepatitis B virus", "HBV", "liver cirrhosis", "hepatic cirrhosis", "liver fibrosis" and "hepatic fibrosis".

INPLASY registration number: This protocol was registered with the International Platform of Registered Systematic Review and Meta-Analysis Protocols (INPLASY) on 05 May 2021 and was last updated on 05 May 2021 (registration number INPLASY202150020).

\section{INTRODUCTION}

Review question / Objective: The central white blood cell populations, neutrophils, and lymphocytes are involved in the pathophysiology of patients with chronic hepatitis B (CHB) infection. We thus performed a meta-analysis of studies investigating the potential link of neutrophil to lymphocyte ratio (NLR) with pathological liver conditions in CHB patients.

Condition being studied: Chronic hepatitis $B$ virus (HBV) infection is a well-established cause of liver-related morbidity and mortality, affecting approximately 240 million people worldwide and results in $\mathbf{8 8 7 , 0 0 0}$ deaths annually (Schweitzer et al., 
2015; Trépo et al., 2014). It is important to note that frequent exacerbations of chronic hepatitis B (CHB) will inevitably result in liver fibrosis (LF) (Fattovich et al., 2008). LF is a dynamic pathological condition that can be slowed down in its initial phases, whereas, without proper clinical management, progressive liver damage may lead to liver cirrhosis (LC). Early LC is reversible, whereas many $\mathrm{CHB}$ patients subsequently progress into decompensated cirrhosis with various complications and ultimately liver failure or primary liver cancer (Hegade et al., 2016; Latief et al., 2018). Therefore, assessing liver function impairment and the exacerbation in CHB patients is essential in clinical practice. Currently, serum aspartate-aminotransferase (AST) and alanine-aminotransferase (ALT) are widely applied in revealing liver function impairment in acute and chronic liver diseases (Hayashi et al., 2015). However, these two diagnostic indexes do not always correlate well with the disease stage and extent of hepato-damage or fibrosis (Kim et al., 2018). Increased serum ALT levels could also be observed once a year in almost $60 \%$ of those patients (Brunetto et al., 2002). Meanwhile, approximately $44 \%$ of hepatitis $B$ e-antigen ( $\mathrm{HBeAg}$ ) negative hepatitis, the ALT remains within the normal range despite disease progression (Brunetto et al., 2002). Importantly, ALT activity does not reflect inflammatory activity in the liver nor the extent of fibrosis in CHB patients (Świderska et al., 2017). The presence of biopsy-proven histologic damage (necroinflammation and fibrosis) is familiar even when ALT is less than two times of upper limit of normal (ULN) in CHB patients (Terrault et al., 2007). Therefore, it is crucial to find potential biomarkers associated with pathological liver conditions in CHB patients. In recent years, several systemic inflammation markers (leucocytes, neutrophils, lymphocytes) have gained significant interest due to their wide availability, low cost, and ability to predict patients' outcomes. Neutrophil to lymphocytes ratio (NLR), calculated from complete blood count with differential count tests, can reveal the immune status and chronic systemic inflammation (Lee et al., 2016; Faria et al., 2016). Peripheral NLR was related to disease progression, predicting liver failure development, and significantly correlated with LF in CHB patients (Fan et al., 2017; Cai et al., 2018). Furthermore, elevated NLR is a potential independent predictor of LF and LC in CHB patients (Kekilli et al., 2015; Biyik et al., 2013).

\section{METHODS}

Participant or population: A total of 19 studies, including 4,494 CHB patients and 1,736 healthy controls (HCs), were finally included.

Intervention: None.

Comparator: None.

Study designs to be included: A comprehensive literature search was conducted by searching PubMed, EMBASE, Web of Science, Cochrane, the Chinese National Knowledge Infrastructure database, and the Wanfang online databases from inception to March 1, 2021. The search keywords were as follows: "neutrophil to lymphocyte ratio", "neutrophil lymphocyte ratio", "neutrophilto-Iymphocyte ratio", "neutrophillymphocyte ratio", "NLR", "hepatitis B", "chronic hepatitis B", "CHB", "hepatitis B virus", "HBV", "liver cirrhosis", "hepatic cirrhosis", "liver fibrosis" and "hepatic fibrosis".

Eligibility criteria: Articles that met the following inclusion criteria were selected: they were cross-sectional or case- control studies related to human studies; assessing the NLR values in CHB patients during the time of admission; providing essential information of patients, especially the number of $\mathrm{CHB}$ patients and controls, and the mean with standard deviation (SD), or median with interquartile range (IQR) of NLR. Exclusion criteria: review article, letters, meeting abstract; duplicate publications or without extractable data.

Information sources: A comprehensive literature search was conducted by 
searching PubMed, EMBASE, Web of Science, Cochrane, the Chinese National Knowledge Infrastructure database, and the Wanfang online databases from inception to March 1, 2021. The search keywords were as follows: "neutrophil to lymphocyte ratio", "neutrophil lymphocyte ratio", "neutrophil-to-lymphocyte ratio", "neutrophil-lymphocyte ratio", "NLR", "hepatitis B", "chronic hepatitis B", "CHB", "hepatitis B virus", "HBV", "liver cirrhosis", "hepatic cirrhosis", "liver fibrosis" and "hepatic fibrosis".

Main outcome(s): A total of 19 studies, including 4,494 $\mathrm{CHB}$ patients and 1,736 healthy controls (HCs), were finally included. Meta-analysis of continuous data indicated that the NLR was significantly higher in the CHB patients than in the HCs (WMD = 0.24, 95\% Cl: 0.03-0.45; $P=0.026$ ); in the CHB patients with liver cirrhosis than the CHB patients without liver cirrhosis (WMD = 0.72, 95\% Cl: 0.49-0.95, $\mathrm{P}<0.001$ ). Besides, the pooled NLR values in the CHB patients with high liver fibrosis (fibrosis score 3-4) were significantly lower than the CHB patients with low liver fibrosis (fibrosis score 1-2) (WMD $=-0.31,95 \% \mathrm{Cl}:-0.10$ to $-0.52 ; P<0.001)$.

Quality assessment / Risk of bias analysis: The funnel plot was asymmetric of $f$ NLR in CHB patients compared with $\mathrm{HCs}$, and the P-value was 0.013 in Begg's test and 0.007 in Egger's test, indicating potential publication bias.

Strategy of data synthesis: All statistical analyses were performed using the STATA software, version 14.0 (STATA Corp.,College Station, TX, USA). Weighted mean difference (WMD) values were reported with $95 \%$ confidence intervals (Cls), and Pvalue $<0.05$ was considered statistically significant. Data presented as a median with IQR were finally converted to mean with SD using the technique described by a previous study (Hozo et al., 2005). We used the $Q$ statistic to test the heterogeneity of studies (the significance level at $P<0.10$ ). The heterogeneity (12) statistic was also calculated ( $12<25 \%$, no heterogeneity; 12 between $25 \%$ and $50 \%$, moderate heterogeneity; 12 between $50 \%$ and $75 \%$, large heterogeneity; and I $2>75 \%$, extreme heterogeneity) (Bowden etal., 2011). Due to the relatively high heterogeneity within studies, we used a random-effect model to calculate the pooled WMD and $95 \% \mathrm{Cl}$. Besides, we used Begg's adjusted rank correlation test and Egger's regression asymmetry test to detect publication bias. P-value > 0.05 for both tests was no significant publication bias. We conducted a sensitivity analysis to investigate an individual study's influence on overall risk estimate by sequentially excluding one study in each step. The Duval and Tweedie "trim and fill" procedure was performed to assess the possible effect of publication bias further.

Subgroup analysis: Given NLR's heterogeneity between the CHB group and healthy controls, subgroup analyses were conducted according to NOS scores, countries, and study types. The results showed that the CHB group's NLR was significantly higher than in healthy controls, especially in the Chinese population $(P=$ 0.029).

Sensitivity analysis: Meta-analysis of NLR in CHB patients compared with HCs:Sensitivity analysis showed that the pooled WMD was not materially changed when each study was sequentially excluded. Meta-analysis of NLR in CHB patients with low fibrosis compared with CHB patients with high fibrosis: Sensitivity analysis indicated that pooled WMD values were not substantially altered when removing any single study, suggesting that the meta-analysis results were stable. Meta-analysis of NLR in CHB patients with LC compared with the CHB patients without LC: Sensitivity analysis showed that the pooled WMD was not materially changed when each study was sequentially excluded.

\section{Country(ies) involved: China.}

Keywords: neutrophil to lymphocyte ratio; pathological liver conditions; chronic hepatitis $B$ patients; a meta-analysis. 
Contributions of each author:

Author 1 - Xianqiang Liu.

Author 2 - Ye,WX.

Author 3 - Fu,H.

Author 4 - Zhu,SR.

Author 5 - Fu,W.

Author 6 - Yinshuo Han.

Author 7 - Qin Wang. 